\title{
Study on Silicothermic Reduction of Dolomite Electrical Resistance Measurement
}

\author{
By Yoshimoto Wanibe*
}

\begin{abstract}
In order to investigate mainly a primary step of the silicothermic reaction, the behavior of the electrical resistance for the briquettes of ferro-silicon alloys and calcined dolomite was measured. The results obtained explain the following phenomena: (1) The primary reaction between silicon and calcium oxide is very fast to produce the calcium silicide phase in the briquette.

(2) The addition of suitable amounts of calcium fluoride may affect the primary reaction and accelerate the overall reaction.

(3) The ferro-silicon alloys with about 75 and $80 \%$ silicon are suitable for the silicothermic reaction. (4) Increasing the briquetting pressure sometimes stifled the progress of the reaction.
\end{abstract}

(Received December 27, 1971)

\section{Introduction}

The reduction of $\mathrm{MgO}$, especially in calcined dolomite as a raw material, by ferro-silicon at high temperature in vacuo has extensively been studied and industrially operated for many years. It has been pointed out that the exothermic reaction between $\mathrm{CaO}$ in calcined dolomite and silicon in ferro-silicon alloys to produce a liquid calcium silicide takes place. In one of the most distinguished studies on the presence and the role of the silicide, Schneider et al. ${ }^{(1)}$ drew the conclusion that the reaction is a primary step in the overall silicothermic reaction and the silicide then affects the reduction to magnesium and $\mathrm{Ca}_{2} \mathrm{SiO}_{4}$.

The formation of this silicide is of greatest importance as a part of the reaction. After some controversial discussions, the $\mathrm{CaSi}_{2}$ would seem to be identified conclusively as the silicide phase. The reaction to form the $\mathrm{CaSi}_{2}$ and its subsequent role, however, remain to be further investigated. Thus, an intensive investigation of the silicothermic reaction, particularly during the beginning stage of the reaction, is highly essential and worthwhile.

In the present work, therefore, much attention is focussed on the primary reaction during the beginning stage of the reaction. The experiment was carried out by measuring the electrical resistance of the briquettes of calcined dolomite and ferro-silicon alloys under various conditions.

\section{Materials and Apparatus}

\section{Materials}

Dolomite in the form of calcined powder was used for this investigation. The chemical composition is shown in Table 1.

Ferro-silicon alloys with compositions ranging from 73 to $86 \%$ silicon used contains about 0.1 manganese and $1 \%$ metallic aluminum. Table 2 shows the analytical results of silicon contents and the screenanalysis data in more details. The alloys were supplied from Dominion Magnesium Limited.

Calcium fluoride used is a Fisher Certified Reagent for chemical analysis. The detailed chemical composition is shown in Table 3.

\section{Briquette preparation}

Calcined dolomite and ferro-silicon alloy, and sometimes calcium fluoride powder, were mixed in a rotating glass jar with a charge of steel balls; normally, $15 \%$ excess ferro-silicon over the stoichiometric requirement was added. According to Etsell's work ${ }^{(2)}$, the following procedure was undertaken to prepare a

Table 1 Composition of calcined dolomite

\begin{tabular}{l|r}
\hline Chemical composition & (wt \%) \\
\hline Loss on ignition & 2.46 \\
Acid insolubles & 0.36 \\
$\mathrm{R}_{2} \mathrm{O}_{3}$ & 0.20 \\
$\mathrm{CaO}$ & 57.80 \\
$\mathrm{MgO}$ & 38.49 \\
$\mathrm{CaO} / \mathrm{MgO}$ & 1.50 \\
\hline
\end{tabular}

Table 2 Silicon contents and screen analysis of ferro-silicon alloys.

\begin{tabular}{c|c|c|c|c|c|c|c}
\hline \multirow{2}{*}{$\begin{array}{c}\text { Silicon content } \\
\text { (wt \%) }\end{array}$} & \multicolumn{7}{|c}{$\begin{array}{c}\text { Screen analysis } \\
\text { (wt \%) }\end{array}$} \\
\cline { 2 - 8 } & $+35 \mathrm{M}$ & $+48 \mathrm{M}$ & $+65 \mathrm{M}$ & $+100 \mathrm{M}$ & $+200 \mathrm{M}$ & $+325 \mathrm{M}$ & $-325 \mathrm{M}$ \\
\hline 73.8 & 1.0 & 3.6 & 10.6 & 16.4 & 30.8 & 14.2 & 23.4 \\
75.7 & 0.1 & 0.7 & 4.6 & 15.4 & 30.2 & 18.8 & 30.4 \\
81.2 & 1.4 & 6.6 & 8.2 & 8.4 & 23.4 & 18.2 & 33.8 \\
85.9 & 1.6 & 6.0 & 12.2 & 17.8 & 26.4 & 15.0 & 21.0 \\
\hline
\end{tabular}

* Department of Metallurgy, Faculty of Engineering,

(2) Etsell: M. A. Sc. Thesis, University of Toronto, (1960). Nagoya University, Nagoya, Japan.

(1) A. Schneider et al.: Z. Erzbergb. Metallhüttenw., 12 (1959), 103, 164, 224.

Trans. JM 
Table 3 Composition of calcium fluoride.

\begin{tabular}{c|l}
\hline Chemical composition & (wt \%) \\
\hline $\mathrm{Ba}$ & 0.005 \\
$\mathrm{Cl}$ & 0.01 \\
$\mathrm{~Pb}$ & 0.005 \\
$\mathrm{Fe}$ & 0.005 \\
$\mathrm{~N}$ & 0.000 \\
$\mathrm{SO}_{4}$ & 0.09 \\
\hline
\end{tabular}

suitable contact between the $\mathrm{Pt}$ plate electrodes and oxide briquettes. $1 \mathrm{~g}$ of the mixture was briquetted in a plunger press of $1 / 2$ inch diameter at $200 \mathrm{~kg} / \mathrm{cm}^{2}$ to keep the faces somewhat rough. Pt black powder was then sprinkled into the one side face. The briquette was re-pressed at $400 \mathrm{~kg} / \mathrm{cm}^{2}$. The other side of the briquette was also covered with $\mathrm{Pt}$ black powder. Finally almost all briquettes were produced at 2000 $\mathrm{kg} / \mathrm{cm}^{2}$ to be about $0.36 \mathrm{~cm}$ in thickness.

In order to investigate the effect of the briquetting pressure on the electrical resistance of the specimens, briquettes were made in the same way as mentioned above except for the final briquetting pressure adjusted to each value.

\section{Apparatus}

The experimental apparatus used in this work consisted of an argon gas purifier, a vacuum system, a temperature-controlled furnace, and an electrical resistance measuring equipment. Figure 1 shows a schematic diagram of the assembled apparatus.

As to the gas purifier, argon gas from a cylinder was passed through a diaphragm pressure regulator, a flow control valve (Brooks Model 8800) and a rotometer (Brooks Model FV-1110), and purified with magnesium perchlorate and ascarite columns. The gas was further purified by a titanium getter furnace being heated at $800^{\circ} \mathrm{C}$. The refined argon gas was then led into the furnace, and a manometer and a safety relief valve were mounted in the way.

The vacuum system was furnished with a rotary pump and a mercury diffusion pump. The pressure was detected by a thermo-couple vacuum gauge (Cenco No. 94178) and a Mcleod gauge (Vacustat Model 2-G).

The temperature of the furnace was controlled by inserting a Chromel/Alumel thermocouple into the furnace wall coupled with a mercury relay switch and a variable resistor. The temperature of the briquette was measured by a $\mathrm{Pt} / \mathrm{Pt}-13 \% \mathrm{Rh}$ thermocouples, the output of which was recorded on an electronic recorder (Phillips Model 110, 740/19) and checked by a potentiometer (Rubicon Instrument Model 2745).

The experiment was carried out in a horizontal resistance furnace equipped with two movable independent heaters, as described in details by Wynnyckyj $^{(3)}$. The details of the assembly unit for measurement of the electric resistance of the briquette is shown in Fig. 2. The briquette coated with Pt black has a closest contact with two Pt electrodes by sin-

(3) J. R. Wynnyckyj: M. A. Sc. Thesis, University of Toronto, (1965).

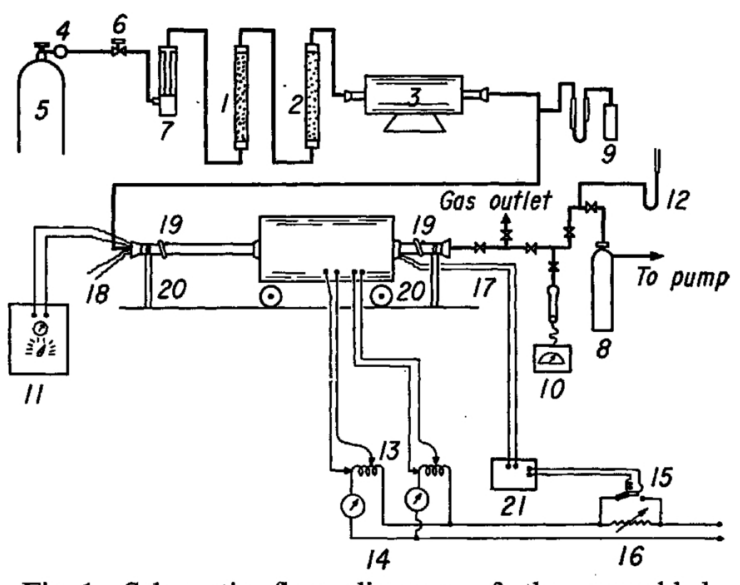

Fig. 1 Schematic flow diagram of the assembled apparatus.

1: magnesium perchlorate column

2: ascarite column

3: titanium getter furnace $\left(800^{\circ} \mathrm{C}\right)$

4: diaphragm pressure regulator

5: argon cylinder

6: flow control valve-Brooks model 8800

7: rotometer $\sim$ precision scientific

8: mercury diffusion pump

9: manometer and safty relief valve

10: thermocouple vacuum gauge-Cenco No. 9418

11: conductivity bridge-COB -12

12: McLeod gauge-Vacustat model 2-G

13: variacs

14: ammeters

15: mercury relay switch

16: variable resistor

17: controller thermocouple

18: charge thermocouple

19: water cooling sleeves

20: furnace tube supports

21: controller

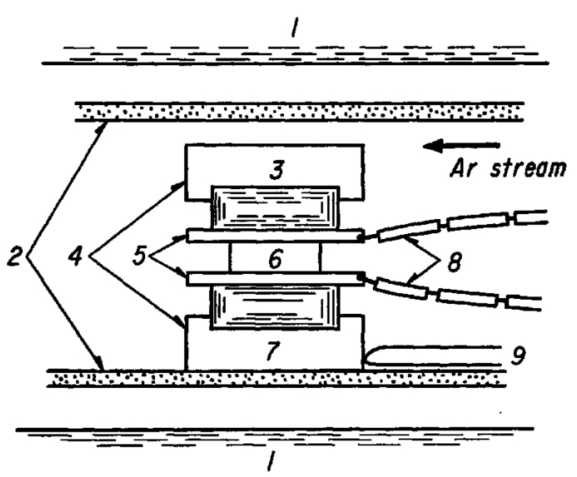

Fig. 2 Cross-sectional diagram of the electric resistance measuring assembly.

1: furnace wall 2: reaction tube

3: weight 4: alumina insulaters

5: platinum electrodes 6: sample

7: sample holder 8: platinum leads

9: thermocouple

tering at high temperature. The electrical resistance of the briquette was measured by a conductivity bridge (The Canadian Research Institute Model COB-12) through two Pt leads welded to the Pt electrodes.

\section{Experimental Procedures}

The resistance measuring unit was assembled and set into an Inconel reaction furnace tube. For experi- 
ments in argon atmosphere, purified argon gas was replaced for air in the Inconel tube and was passed through the charge at a rate of $50 \mathrm{cc} / \mathrm{min}$ throughout the run. In the experiment being carried out in vacuo, the vacuum pumps were operated to suck out the air from the measuring assembly. After the furnace was moved to the farthest position from the measuring assembly, the furnace temperature was raised at a given temperature. Then, as soon as the first values of the electric resistance and the temperature of the specimen were measured as the starting point, the furnace was moved to set the measuring unit into the hot uniform temperature zone of the furnace and to heat the specimen as rapidly as possible up to a given temperature. Then these measurements were continued every minute. Even though the temperature of the specimen was raised as rapidly as possible, it took about $20 \mathrm{~min}$ for the temperature to reach a given point when the controller was turned on to maintain a constant temperature as shown in Fig. 3.

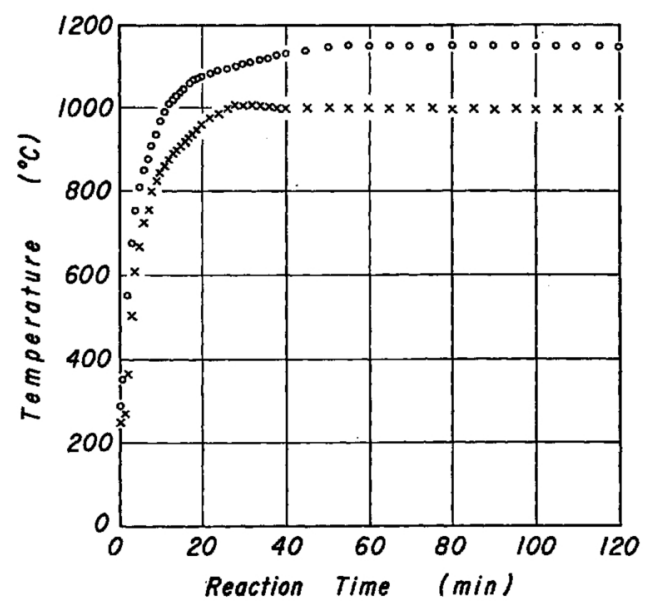

Fig. 3 Time-temperature curve.

\section{Experimental Results}

The time-dependency of the electrical resistance of the briquette was determined at 1000, 1050, 1100 and $1150^{\circ} \mathrm{C}$ under a stream of purified argon gas. The results are summarized in Fig. 4, where the relation between the reaction time and the specific resistance is shown. The specific electrical resistances were calculated by using the diameter and the thickness of the pressed briquettes. A dramatic decrease in resistances occurred until the reaction time of about 4 to $7 \mathrm{~min}$ has elapsed. At this point a minimum is reached where the temperatures of the specimens is about 750 to $780^{\circ} \mathrm{C}$. Then the specific resistance increased rapidly for about 3 to $5 \mathrm{~min}$ until the briquettes temperatures reached about 870 to $930^{\circ} \mathrm{C}$. Then, the electric resistance of the briquettes behaved to draw its own characteristic curves including several kinks, knees, arrests, etc., depending upon the experimental temperature. At the final period of the behaviors, all the resistances increased gradually. According to this figure, the rise in the experimental temperature decreased the final specific resistance value of the

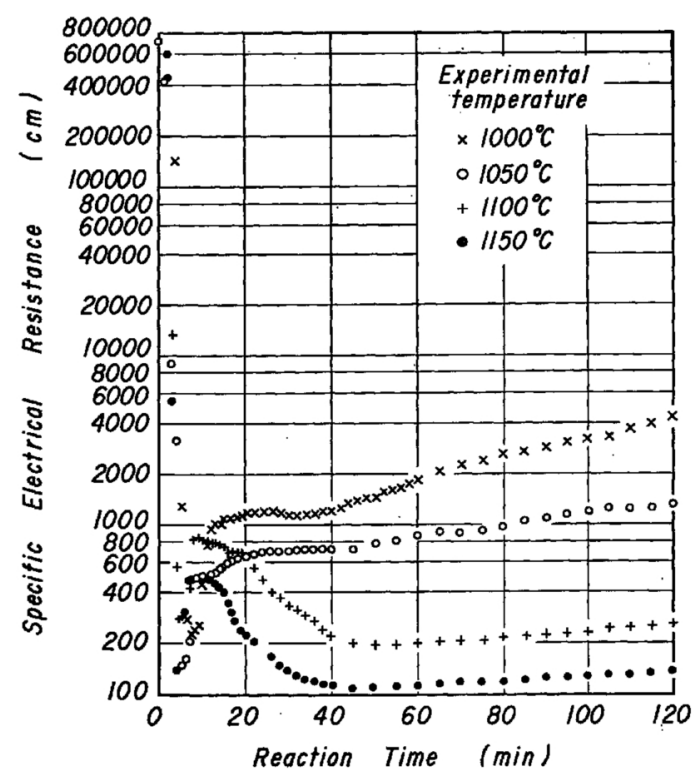

Fig. 4 Time-dependency of specific electrical resistance; argon gas.

briquette. Furthermore, this figure shows that the experiments at 1000 and $1050^{\circ} \mathrm{C}$ has a quite similar tendency in the behaviors of electrical resistivity, while those at 1100 and $1150^{\circ} \mathrm{C}$ also have another tendency, even though each resistivity has its own different value.

The measurement of specific resistivity was also carried out in vacuo at $1000,1050,1100$ and $1150^{\circ} \mathrm{C}$. The results are shown in Fig. 5. In the early stage of the experiments the specific resistance versus reaction time curves are sharply fluctuated for a period of several minutes. The specific resistance of the specimen first went down sharply until the reaction time elapsed about 3 to $6 \mathrm{~min}$ and reached a minimum value when the briquette temperature rose up to about 690 to $720^{\circ} \mathrm{C}$. Then the temperature rose for about $1 \mathrm{~min}$ until it reached $780^{\circ} \mathrm{C}$ from about $740^{\circ} \mathrm{C}$, and attained a maximum value. Again, it decreased rapidly

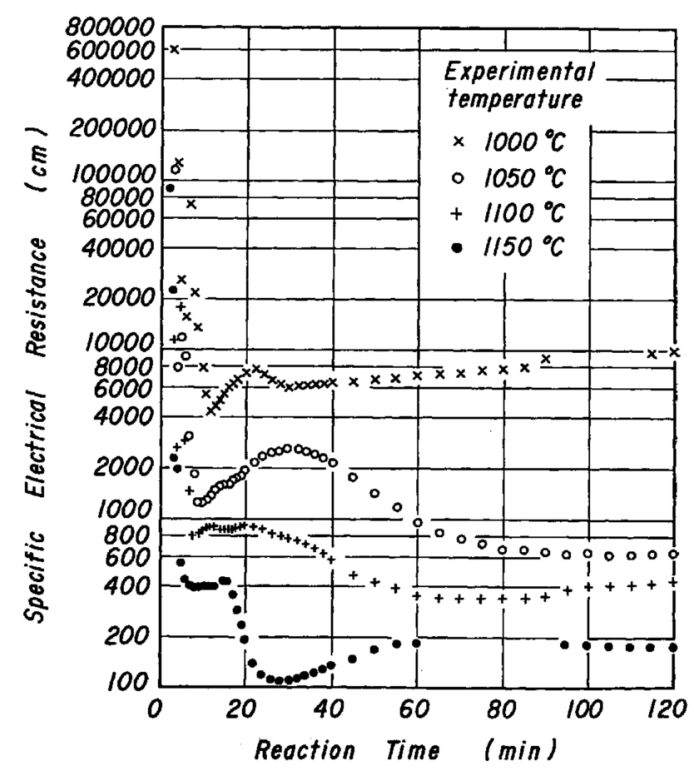

Fig. 5 Time-dependency of specific electrical resistance; vacuum. 
until the reaction time reached about 8 to $12 \mathrm{~min}$, and became a minimum value when the briquette temperature was between about 880 and $910^{\circ} \mathrm{C}$. Then the resistivity of the briquette (traced its) own characteristic curve including the several kinks, which correspond to the experimental temperatures and increased gradually in the final stage. As shown in Fig. 5, the increase in the experimental temperature decreased the final specific resistance having a tendency similar to that under the argon atmosphere. However, it is shown that the results of experiments in vacuum at 1000,1050 and $1100^{\circ} \mathrm{C}$ have a similar tendency in the time dependence of the specific electric resistance for the briquettes, except for that at $1150^{\circ} \mathrm{C}$. From a comparison of Figs. 4 and 5 , it is seen that the fluctuation of the resistance versus time curve measured in vacuum occurred at the earier reaction time, with a severer and sharper ways than that obtained under refined argon gas. In almost all cases the specific resistance values during the fluctuation period in vacuum were also higher than those under the argon stream at the same temperature. It appears also that the silicothermic reaction can proceed much faster in the vacuum condition than that in the purified argon gas.

The effect of the silicon content in the ferro-silicon alloy on the silicothermic reaction was investigated at $1150^{\circ} \mathrm{C}$ in consideration of the difference between the experimental results obtained in argon and vacuum. Figure 6 shows the effect when the experiment was carried out under the stream of refined argon gas. The experimental results in vacuo are also shown in Fig. 7. According to these figures, about 75 and $80 \%$ silicon contents in the ferro-silicon make the reaction progress rapidly, compared with the other ferro-silicon alloy of about $85 \%$ silicon; this tendency was very

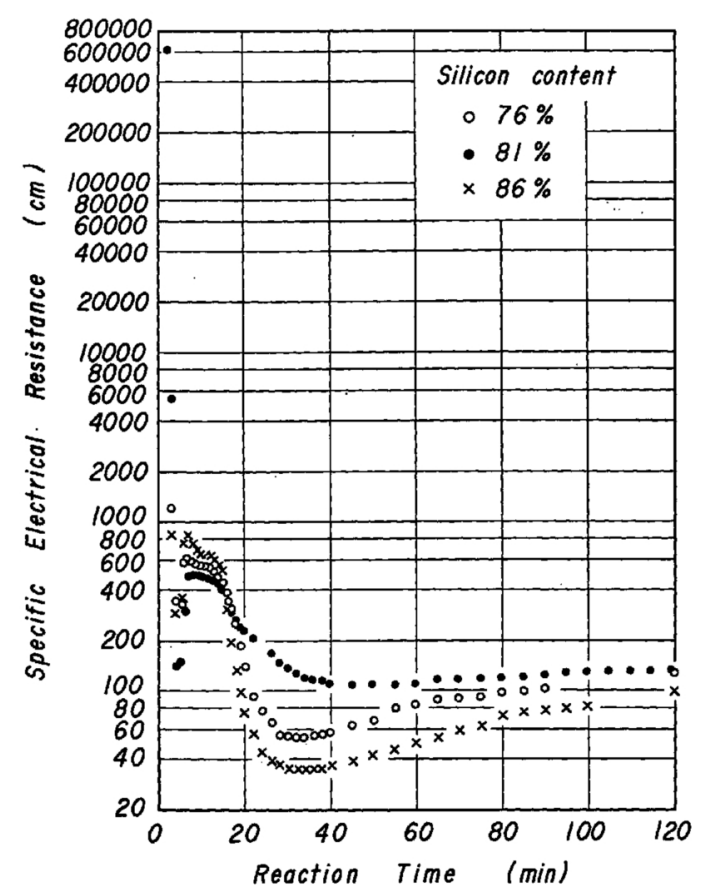

Fig. 6 The effect of the silicon content in ferro-silicon alloys on the reaction; argon gas.

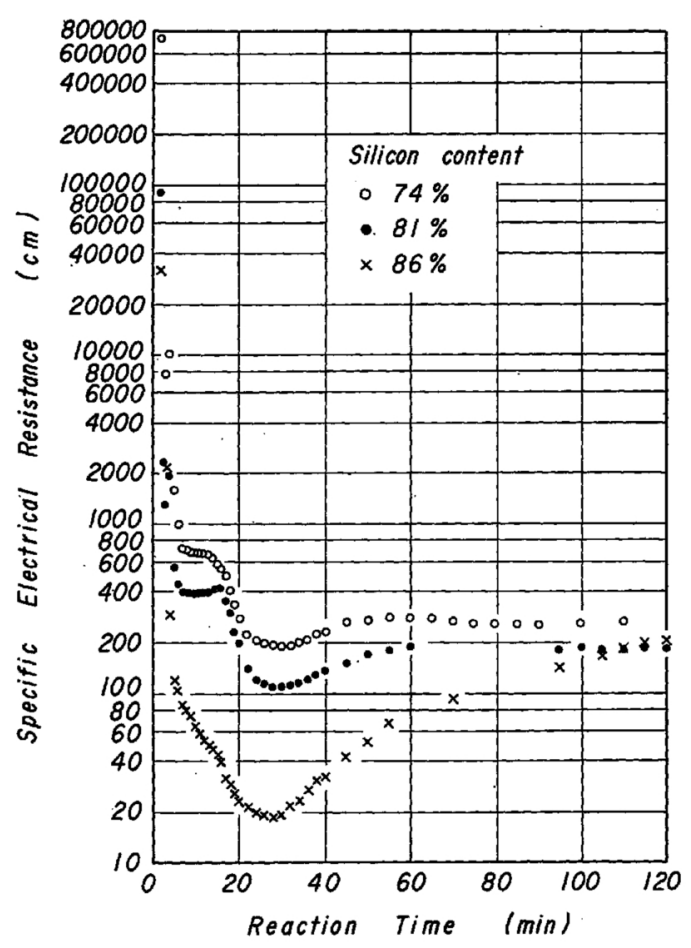

Fig. 7 The effect of the silicon content in ferro-silicon alloys on the reaction; vacuum.

remarkable in the experiment carried out in vacuum.

Next, the effect of the final briquetting pressure on the reaction between the calcined dolomite and the ferro-silicon alloy was investigated by using the ferro-silicon of about $80 \%$ silicon at $1150^{\circ} \mathrm{C}$ under the argon atmosphere. The results are shown in Fig. 8. Increasing the briquetting pressure decreased the resistivity in the final stage and brought about a larger fluctuation of the resistivity, particularly in the case of the final briquetting pressure of $7 \mathrm{t} / \mathrm{cm}^{2}$.

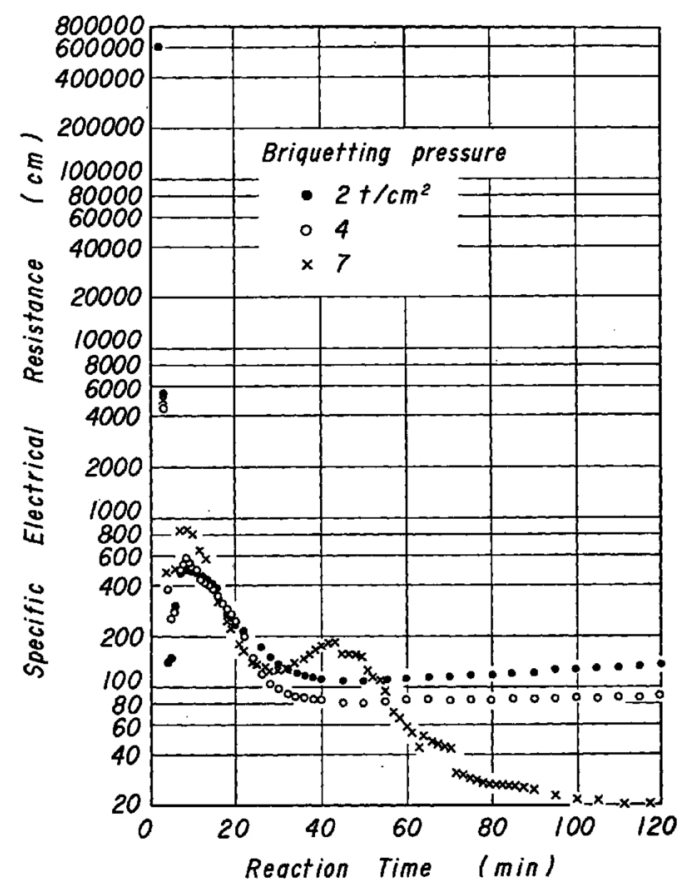

Fig. 8 The effect of the final briquetting pressure on the reaction; argon gas. 


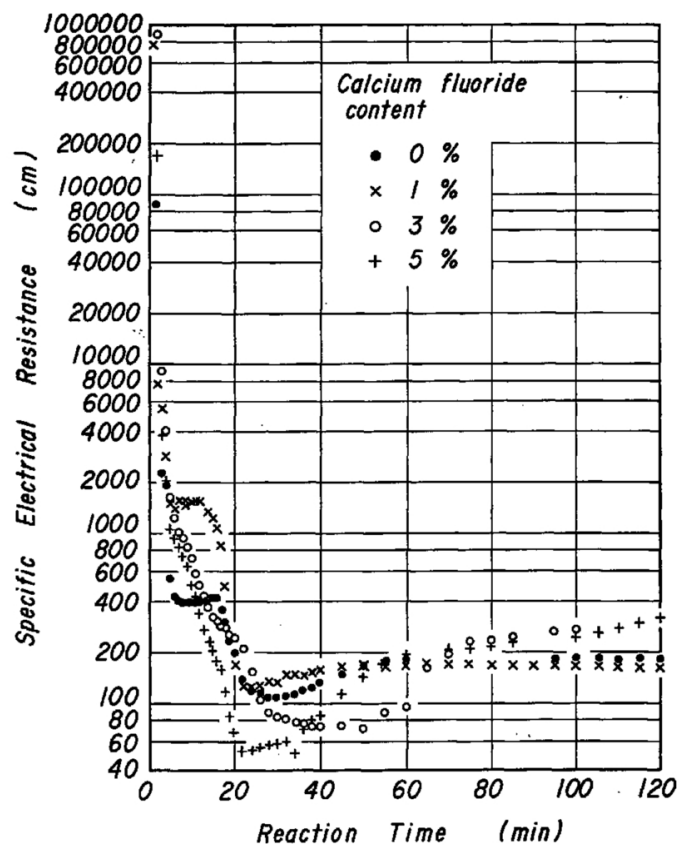

Fig. 9 The effect of the calcium fluoride on the reaction; vacuum.

According to the references ${ }^{(4)}$, small additions of calcium fluoride to the briquette of calcined dolomite and ferro-silicon alloys give a catalytic effect to the reactions. This effect was investigated by adding calcium fluoride of about 1,3 and $5 \%$ to a mixture of the dolomite and ferro-silicon alloys at $1150^{\circ} \mathrm{C}$ in vacuum. The results are shown in Fig. 9. This figure shows that the addition of calcium fluoride leads to no "knee-shape" fluctuation in the time-dependent curve of the electrical resistance and continuously decreases the resistance up to about $12 \mathrm{~min}$ of the reaction time, except for the experiment carried out by using the briquette sample containing about $1 \%$ calcium fluoride. A similar behavior of the time-dependency of the resistance was seen between the experiments carried out by using the briquettes containing about 3 and $5 \%$ calcium fluoride, while a different similar tendency was seen between the cases of about $1 \%$ and zero calcium fluoride. The ascent of the curve after passing through the minimum value indicates that the addition of calcium fluoride to the briquettes accelerates the evolution of magnesium.

\section{Discussion}

An attempt was made to analyze the present experimental results in connection with the process of the silicothermic reaction of dolomite. Many reaction mechanisms have so far been postulated to explain such a rapid reaction rate and a wide discrepancy between the experimental results and the thermodynamic calculation data on the equilibrium pressure of magnesium vapor over the mixtures of calcined dolomite and ferro-silicon alloys ${ }^{(1)(3) \sim(5)}$.

(4) L. M. Pidgeon and J. A. King: Discuss. Faraday Soc., 4 (1948), 197.

(5) J. M. Toguri and L. M. Pidgeon: Can. J. Chem., 40 (1962), 1765.
At present it seems certain that a liquid calcium silicide phase, $\mathrm{CaSi}_{2}$, is produced by the primary step of the overall silico-thermic reaction.

Matsushima et al. ${ }^{(6)}$ have investigated the silicothermic reaction under the argon gas stream by using a differential thermal analysis technique. According to their experiments, the lowest temperature for the formation of calcium silicide is $550^{\circ} \mathrm{C}$, and the reaction becomes exothermic until the temperature reaches to about $950^{\circ} \mathrm{C}$. Above $950^{\circ} \mathrm{C}$ a large endothermic peak is observed. This endothermic phenomenon has been defined as the indication of the melting of calcium silicide.

In the present work, discontinuity in the timedependent curve of the electrical resistance seems to be a clue to investigate this reaction. Hence, particular attention was drawn to these fluctuations in the curves and to their interpretation.

First of all, the present results were discussed by using the phase diagram of the $\mathrm{Ca}-\mathrm{Si}$ system. Wynnyckys et al. ${ }^{(3)}$ examined this binary system in detail by extensive metallographic, X-ray and microprobe studies in addition to the vapor pressure measurements, and demonstrated that the presently accepted phase diagram for this system as shown in Fig. $10^{(7)}$ is incomplete and there are two additional intermetallic phases. The peritectic melting point of $1020^{\circ} \mathrm{C}$ shown in the accepted diagram corresponds to one of these compounds, $\mathrm{Ca}_{3} \mathrm{Si}_{4}$. However, since it is still necessary to confirm the presence of these compounds and to make clear of the relation between the phases, the acceptable phase diagrams were employed here.

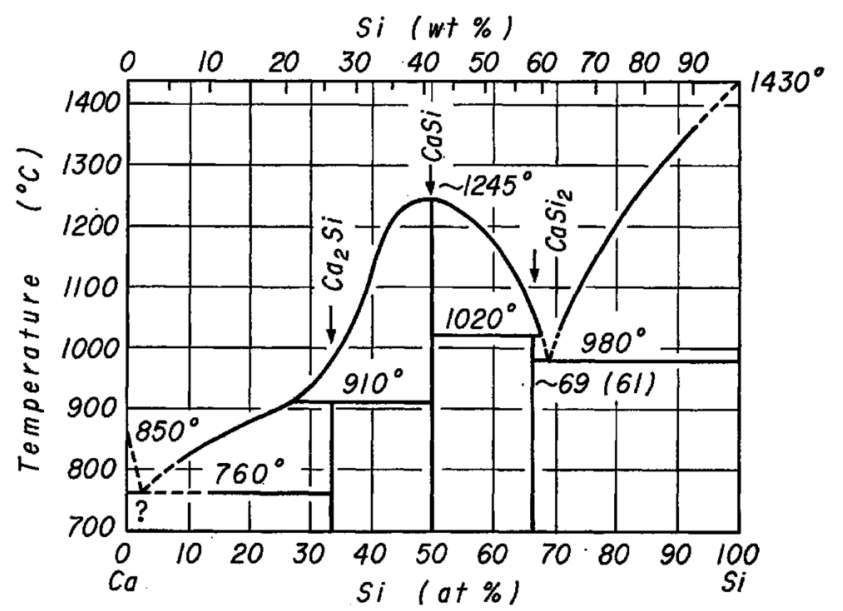

Fig. 10 Equilibrium diagram for the calcium-silicon system.

During the initial stage of the silico-thermic reaction, the following phenomena are expected to occur, according to the coupled factors between the rates of formation of calcium silicide and the rising temperature of the briquettes:

(1) The primary reaction between the calcium oxide in the calcined dolomite and the silicon in the

(6) T. Matsushima and L. M. Pidgeon: Private Reference.

(7) M. Hansen: Constituation of Binary Alloys, 2nd Ed., McGraw Hill, (1958). 
ferro-silicon alloy is not completed, before the temperature of the briquette reaches the eutectic $\left(980^{\circ} \mathrm{C}\right)$ and liquidus temperatures. In this case, certain irregularities in the specific resistance are expected to occur at the eutectic and liquidus temperatures. The liquidus temperature depends upon the rate of reaction.

(2) The preliminary reaction is so fast that the production of calcium silicide is completed far below the eutectic temperature, where the irregularities in the time-dependent curve of the resistivity are expected to take place at the peritectic $\left(1020^{\circ} \mathrm{C}\right)$ and liquidus temperatures (about $1050^{\circ} \mathrm{C}$ ).

(3) This is the case in which the above two cases are combined. With increases in temperature, the rate of the formation of calcium silicide appears to becomes faster. Hence the composition of the calciumsilicon alloy produced can be expected to be more calcium-rich. Therefore, there is a possibility that the eutectic, peritectic and melting transformations occur during the reaction process with the increase in the temperature of the briquette.

In the expectation of one of the above three phenomena, the irregularities in the time-dependency of the electrical resistance in the present results were examined, even though the results are not enough capable of determining distinctly such phenomena. The experimental results either under the refined argon gas atmosphere or in vacuo, have shown only the eutectic transformation. In the experiment under the refined argon gas flow at $1050^{\circ} \mathrm{C}$ the phenomenon (1) was observed, but the results at the same temperature in vacuum showed the phenomenon (2). The experiments at higher temperatures, 1100 and $1150^{\circ} \mathrm{C}$, under both conditions showed the phenomenon (2).

Therefore, in the condition of the present work the preliminary reaction seems to be very fast and to complete in about half an hour at relatively high temperatures.

The silicothermic reaction has been studied comprehensively by Schneider et al. ${ }^{(1)}$ One of their experiments was carried out by measuring the electrical resistance of the briquette of calcined dolomite and ferro-silicon alloy. As their experimental temperatures were lower and the briquetting pressure and the silicon content in the ferro-silicon alloys used were higher than those in the present work, a direct comparison between both results was impossible. However, since the same principle as in the present study was used in their study, the comparison the phenomena observed would be most interesting.

The following common phenomena were observed between the experimental results by the present author and Schneider et al.

(1) During the final stage of the silico-thermic reaction, the electrical resistance of the briquette increased gradually under argon stream and in vacuum. This phenomenon can be explained by the increase in porosity of the specimen due to the vaporization of magnesium as the secondary reaction proceeds.

(2) The final electrical resistances obtained in vacuo and in argon gas atmosphere approach the same values which depend upon the factors such as the temperature and briquetting pressure, even though each value showed its own practically modified behavior in the curve.

(3) The increase in briquetting pressure resulted in a decrease in electrical resistance and a larger fluctuation in the time-dependency of electrical resistance during the beginning stage, even though the present experiment was carried out under the refined argon gas stream and not in vacuum as in the experiment of Schneider et al. This phenomenon shows a non-uiform progress of the reaction. Since this fluctuation took place during the relatively later stage of the reaction, there seems to be a certain obstacles, such as the choke of the magnesium vapors, in the secondary reaction.

There are some differences in the phenomena observed in the present work and by Schneider et al.:

(1) In the study of Schneider et al. fluctuations in the time-dependency of the electrical resistance, which have been ascribed to the vaporization of rest-water and carbon dioxide from specimen, were reported to occur only in vacuum and not in argon atmosphere. However, the present experimental results show that these fluctuations take place not only under the vacuum condition, but also under the argon stream.

(2) Compared with the results of Schneider et al., the present results show that the decrease in specific electrical resistance during the beginning stage of the reaction is very sharp.

(3) Schneider et al. have also reported that the briquette treated at high temperatures above $1000^{\circ} \mathrm{C}$ is very hard. However, in the present work the specimen subjected to a relatively low briquetting pressure is not very hard and remains so after the treatment even at high temperatures.

\section{Summary}

In the present work the electrical resistance of the briquette of calcined dolomite and ferro-silicon alloys was measured with the conductivity bridge, and the silicothermic reaction was investigated. Careful examination of the results obtained during the beginning stage of the reaction led to the following conclusions:

(1) The initial reaction, which occurs between silicon in the ferro-silicon alloy and calcium oxide in calcined dolomite to produce a calcium silicide alloy, the actual reducing agent, proceeds so fast that the composition of the alloy seems to reach soon an intermetallic phase, $\mathrm{CaSi}_{2}$. This is accelerated at higher temperatures and also in vacuum rather than under the refined argon stream.

(2) Additions of about 3 and $5 \%$ calcium fluoride to the briquettes bring about some changes in the primary reaction and accelerate the evolution of magnesium, while the addition of about $1 \%$ calcium fluoride does not.

The following phenomena were also confirmed from the results obtained under the various condi- 
tions:

(3) The ferro-silicon alloys including about 75 to $80 \%$ silicon increase the rate of the silicothermic reaction. This tendency is clearer in vacuum condition.

(4) Increasing the briquetting pressure seems to cause stifling the progress of the reaction.

\section{Acknowledgments}

The author wishes to express his gratitude to Dr.
L. M. Pidgeon for his valuable suggestions and guidance throughout the present work. He is also indebted to Dr. J. M. Toguri for his help in preparing this report. Thanks are also due to Dominion Magnesium Company for suppling the calcined dolomite and the grounded ferro-silicon alloy. This research was supported by the NRC scholarship. 